\title{
Linardatos, Dimitros, Autonome und vernetzte Aktanten im Zivilrecht. Grundlinien zivilrechtlicher Zurechnung und Strukturmerkmale einer elektronischen Person
}

\author{
Mohr Siebeck Verlag, Tübingen, 2021. XXII, 652 Seiten. ISBN \\ 978-3-16-160756-1
}

\section{Christian Armbrüster}

Angenommen: 6. Januar 2022 / Online publiziert: 14. Januar 2022

(C) Der/die Autor(en) 2022

Die fortschreitende Digitalisierung hat auch für das Versicherungsrecht längst zu völlig neuartigen Fragestellungen geführt. Je mehr dank Künstlicher Intelligenz (KI) Algorithmen an die Stelle von menschlichem Handeln treten, umso dringlicher stellt sich die Frage, wer für das entsprechende Verhalten und dessen Folgen verantwortlich ist. Dies gilt etwa für mittels KI erstellte Vertragserklärungen oder für autonom gesteuerte Fahrzeuge. Linardatos hat es sich in seiner Mannheimer Habilitationsschrift zum Ziel gesetzt, der Frage nach der rechtlichen Anerkennung einer elektronischen Person (e-Person) als ,,artifizielles Rechtssubjekt“ (S. 184) nachzugehen. Dabei eröffnen sich, worauf im Folgenden zurückzukommen ist, auch für den versicherungsrechtlich interessierten Leser interessante Themenfelder.

Bereits im Titel der Arbeit greift der Autor mit dem Wort „Aktanten“ einen Begriff auf, der seinen Ursprung in der Soziologie hat und mit dem dort gesellschaftlich und wirtschaftlich bedeutsames Verhalten innerhalb eines sozialen Systems benannt wird. Im hier interessierenden Kontext zählt Linardatos zu den autonomen und vernetzten Aktanten insbesondere Roboter, Softwareagenten, Bots (als eine Untergruppe der Softwareagenten) sowie Multiagentensysteme (S. 28 ff.).

Was die durch das Handeln von Aktanten im solchermaßen verstandenen Sinne aufgeworfenen Rechtsfragen angeht, so wendet der Autor sich zunächst der allgemeinen Rechtsgeschäftslehre zu (S. $99 \mathrm{ff}$.). Dabei gelangt er über eine analoge Anwendung der Stellvertretungsregeln ( $\S \S 164 \mathrm{ff}$. BGB) zu im Wesentlichen sachgerechten Zurechnungen. Für die Anerkennung einer e-Person als Rechtssubjekt ergibt sich daraus nach verbreiteter, auch vom Autor (S. 181, 183) im Grundsatz geteilter Ansicht kein zwingender Bedarf. Wenn er gleichwohl meint, eine solche Anerkennung sei geboten, soweit dies ,einem im gesellschaftlichen Verständnis ma-

Christian Armbrüster $(\bowtie)$

Fachbereich Rechtswissenschaft, Freie Universität Berlin, Van't-Hoff-Str. 8, 14195 Berlin,

Deutschland

E-Mail: c.armbruester@fu-berlin.de 
nifestierten Realitätsbezug am besten zu entsprechen vermag“ (S. 183), bleibt dies nicht nur aus einer anwendungsorientierten Perspektive allzu sehr im Ungefähren.

Ungleich komplexer ist die Frage, ob eine e-Person geschaffen werden soll, im Haftungsrecht. Dabei erwähnt Linardatos (S. 184) eingangs das Autonomie- und das Vernetzungsrisiko als Herausforderungen. Im Vertragsrecht plädiert er für eine Zurechnung analog § 278 BGB (S. 205 ff.). Damit lasse sich die Herstellerhaftung begrenzen; zugleich seien Autonomierisiken erfassbar und es werde eine Verhaltenssteuerung erreicht. Auch für diese Lösung bedarf es, wie der Autor (S. 264) wiederum selbst erkennt, keiner Anerkennung einer e-Person. Letzteres gilt auch für die außervertragliche Haftung (S. 411). Bei ihr hält der Autor die bestehenden spezialgesetzlichen Tatbestände einer Gefährdungshaftung, die er im Wege einer Rechtsanalogie auf die Haftung für autonome und vernetzte Systeme übertragen will, für ausreichend. Gleichwohl meint er einen bedeutenden Anwendungsbereich für die Anerkennung einer e-Person als „Knotenpunkt der Akteure“ (S. 411) und als Vehikel zur effizienten Verteilung von Schadenslasten zu erkennen.

Was die Verbundenheit von Nutzern einer Blockchain betrifft, so geht der Autor auf die verschiedenen Erklärungsmodelle ein, insbesondere auf BGB-Gesellschaft und Bruchteilsgemeinschaft. Dabei bringt er jeweils gewichtige Einwände vor. Nicht zu überzeugen vermag es freilich, wenn Linardatos für die GbR einen entsprechenden Rechtsbindungswillen ungeachtet der insoweit bestehenden geringen Anforderungen verneint (S. $422 \mathrm{ff}$.), während er diesen für die Bruchteilsgemeinschaft bejaht (,kleiner gedanklicher Kniff“; S. 453). Freilich hält der Autor trotz deutlich erkennbarer Sympathie für die Anwendung der Regeln über die Bruchteilsgemeinschaft auch bei der Blockchain letztlich wiederum die e-Person für vorzugswürdig (S. 464 ff.).

Nachdem solchermaßen der argumentative Boden für eine Anerkennung der e-Person bereitet ist, legt der Verfasser deren Strukturmerkmale dar, um daraus sodann einen ausführlichen Gesetzesvorschlag zu erarbeiten. Kernelement ist eine Kombination von Konzessions- und Normativsystem (S. 522 ff.). Die besonders kritische Frage nach Kapitalaufbringung und Haftungsvermögen veranlasst Linardatos dazu, entweder Regeln zur Kapitalaufbringung und Vermögensbindung oder (in Anlehnung an $\S 8$ Abs. 4 S. 1 PartGG) eine Versicherungslösung in den Raum zu stellen. Beide Lösungen haben Vor- und Nachteile, welche der Autor näher schildert. So fehlt bei der Versicherungslösung für die e-Person anders als bei einer Partnerschaftsgesellschaft die Möglichkeit, bei Leistungsfreiheit des Versicherers (etwa wegen Obliegenheitsverletzung oder Prämienzahlungsverzugs) ohne Weiteres auf andere Haftungssubjekte zugreifen zu können. Linardatos (S. 544f.) erwägt insoweit einen Schutz von Haftungsgläubigern nach dem Muster der Regeln zur Pflichtversicherung einzuführen. Bei der Eigenkapitallösung hingegen ergibt sich das Problem, dass das ,unbedingt haftende Kernkapital“ (S. 546) nicht zum Wirtschaften eingesetzt werden kann. Aus Sicht des Autors (S. 546f.) relativiert sich dieser Einwand dadurch, dass die konkrete Gestaltung des Rechtssubjekts insoweit Spielräume eröffnen kann.

Der Autor hält die Versicherungs- und die Eigenkapitallösung für ,,prinzipiell gegeneinander austauschbar“ (S. 548). Dementsprechend unterbreitet er hinsichtlich des Haftungsfonds zwei alternative Gesetzesvorschläge. Das Versicherungskonzept 
(S. 573f.) sieht eine Pflichtversicherung vor. Diese ist inhaltlich weitgehend an den Regelungen in $\S 8$ Abs. 3 PartGG und in $\S 51$ Abs. 1 BRAO orientiert. Hinzu kommen eine optionale „Haftungsbeteiligung“ (S. 573) des rechtsfähigen autonomen Aktanten sowie ein Entschädigungsfonds. Nach der Vorstellung des Autors kann der Gesetzgeber das Versicherungskonzept auch mit einem Eigenkapitalkonzept verbinden. Bei Letzterem ist eine Pflicht von Initiatoren und Teilhabern zur Schaffung von Eigenmitteln der e-Person vorgesehen (S. 574).

Insgesamt bietet die Arbeit die bislang umfassendste Abhandlung zur e-Person auf weitgehend aktuellem Stand von März 2021. Den Entwurf eines Artificial Intelligence Act der Europäischen Kommission (COM(2021) 206 final) vom 21.04.2021 mit seinem risikobasierten Ansatz konnte freilich ebenso wie die daran anschließende kontroverse Diskussion nicht mehr berücksichtigt werden. Indessen tut dies der grundlegenden Bedeutung der Arbeit keinen Abbruch. Zwar wird, wer bislang davon ausging, dass sich die von Linardatos angesprochenen Praxisfragen weiterhin durch eine sachgerechte Anwendung und maßvolle richterliche Fortentwicklung der geltenden Regeln bewältigen lassen, nach der Lektüre nicht zwangsläufig die Schaffung einer e-Person befürworten. Dies gilt umso mehr, wenn man sich die Komplexität des umfangreichen Gesetzesvorschlags und die bei einer Umsetzung absehbaren Folgeprobleme - die auch der Autor keineswegs verkennt - vergegenwärtigt. Indessen erscheint mancher Einwand, etwa im Hinblick auf den fehlenden Haftungsfonds einer e-Person, dank der umsichtigen und wohlausgewogenen, nie einseitigen oder gar eifernden, Argumentation von Linardatos nunmehr durchaus in einem anderen Licht. Überdies vermag nicht zuletzt der versicherungsrechtlich interessierte Leser aus der Gegenüberstellung von Eigenkapital- und Versicherungskonzept wertvolle Anregungen zu entnehmen.

Funding Open Access funding enabled and organized by Projekt DEAL.

Open Access Dieser Artikel wird unter der Creative Commons Namensnennung 4.0 International Lizenz veröffentlicht, welche die Nutzung, Vervielfältigung, Bearbeitung, Verbreitung und Wiedergabe in jeglichem Medium und Format erlaubt, sofern Sie den/die ursprünglichen Autor(en) und die Quelle ordnungsgemäß nennen, einen Link zur Creative Commons Lizenz beifügen und angeben, ob Änderungen vorgenommen wurden.

Die in diesem Artikel enthaltenen Bilder und sonstiges Drittmaterial unterliegen ebenfalls der genannten Creative Commons Lizenz, sofern sich aus der Abbildungslegende nichts anderes ergibt. Sofern das betreffende Material nicht unter der genannten Creative Commons Lizenz steht und die betreffende Handlung nicht nach gesetzlichen Vorschriften erlaubt ist, ist für die oben aufgeführten Weiterverwendungen des Materials die Einwilligung des jeweiligen Rechteinhabers einzuholen.

Weitere Details zur Lizenz entnehmen Sie bitte der Lizenzinformation auf http://creativecommons.org/ licenses/by/4.0/deed.de. 